\title{
LV-PP-4-6
}

\section{Laparoscopic right posterior sectionectomy (LRPS) in semi prone position: surgical aspects and results}

\author{
Nikolay BELEV ${ }^{* 1,2}$, Panche KRASTEV ${ }^{1}$, Radoslav PENKOV ${ }^{1}$, Mihail SLAVCHEV \\ 'UMHAT-Eurohospital Ploudiv \\ ${ }^{2}$ U2Surgical Department, Medical Training and Simulation Center, Medical Uiniversity-Plovdiv, Bulgaria
}

Introduction: Laparoscopic hepatectomy has been accepted widely due to its advantages as a minimally invasive surgery, but laparoscopic right posterior sectionectomy (LRPS) has rarely been reported. Tumors located over the right posterior section are considered to be difficult for laparoscopic resection. The Glissonean approach is our technique of choice. The aim of this work is to present our experience in LRPS in semi prone position and to show surgical technical aspects.

Methods: For six years period we were performed 12 LRPS procedures. Patient's data were prospectively collected. Demographics data, tumor characteristics, operative data, and postoperative outcome were collected and analyzed. There were 8 patients with colorectal liver metastasis, 2 HCC, one melanoma metastasis and one breast cancer liver metastasis.

Results: During the period of 2014-2020, we performed 12 LRPS. Median operative time was 200 minutes, median blood loss was 300 $\mathrm{ml}$. Significant bleeding occurred in 1 cirrhotic liver patients. The median size of the tumor resected was $3.5 \mathrm{~cm}$, and the median resection margin was $7 \mathrm{~mm}$. Nine patients show single tumor nodule, 2 patients had two metastases and 1 with 3 metastases. There was no postoperative mortality. Median hospital stay was 6 days.

Conclusions: LRPS is a technically demanding procedure. LRPS in semi prone position is feasible and safe. Semi prone position offers a maximum amount of working space. A proper inflow control is mandatory for excellence of anatomical resection. The Glissonean approach allows safe and masse control of the portal structures of the posterior sector and resulting in less blood loss during transection. 